\title{
Complicated esophageal varicose bleeding and myocardial infarction
}

\author{
Talat Ayyıldız $^{\mathrm{a} *}$, Elif Çolak ${ }^{\mathrm{b}}$, Uğur Arslan, Ŏguzhan Yücel ${ }^{\mathrm{c}}$ \\ a Department of Gastroenterology, Samsun Education and Research Hospital, Samsun, Turkey \\ ${ }^{b}$ Department of General Surgery, Samsun Education and Research Hospital, Samsun, Turkey \\ ${ }^{c}$ Department of Cardiology, Samsun Education and Research Hospital, Samsun, Turkey
}

\section{ARTICLE INFO}

\section{Article History}

Received $\quad 10 / 01 / 2013$

Accepted $\quad 12 / 03 / 2013$

\section{* Correspondence to:}

Talat Ayyıldız

Department of Gastroenterology,

Samsun Education and Research Hospital,

Samsun, Turkey

e-mail: talatayy@gmail.com

\section{Keywords:}

Cirrhosis

Esophageal varices

Myocardial infarction

Terlipressin

\section{ABSTRACT}

\begin{abstract}
Variceal bleeding is one of the most important complications in the patients with liver cirrhosis and also has high morbidity and mortality. For this reason, primary prophylaxis and treatment modality of the variceal bleeding are very important. Many clinical and physiological factors are useful in predicting the risk of variceal bleeding in patients with cirrhosis. In this case report, an esophageal varice bleeding in a patient with end-stage decompensated cryptogenic liver cirrhosis requiring 62 units of blood transfusion and its complications were discussed. Especially, the relation with myocardial infarction (MI) developing after bleeding was evaluated.
\end{abstract}

J. Exp. Clin. Med., 2013; 30:183-185

This report was presented as a poster in 29th Turkish Gastroenterology Week (November 20-25, 2012, Antalya, Turkey)

(C) 2013 OMU

\section{Introduction}

Variceal bleedings are implicated in $50-60 \%$ of upper gastrointestinal bleeding in patients with cirrhosis (Dave et al., 1983; Odelowo et al., 2002). In a variceal bleeding, main targets of therapy are hemodynamical resuscitation, prevention and management of complications and cessation of bleeding. In this report, a case of liver cirrhosis and hepatocellular cancer (HCC) who did not undergo any treatment previously, and who had severe recurrent variceal bleeding and subsequently complicated by spontaneous bacterial peritonitis, hepatic encephalopathy and myocardial infarction was presented.

\section{Case}

A 67 years old male patient who had Child-Pugh Score class C (Child's C) cryptogenic liver cirrhosis and diagnosed with HCC was referred to our hospital from another center where he was followed for six days for esophageal variceal bleeding. At presentation, blood pressure, pulse, haemoglobin values were $60 / 40 \mathrm{mmHg}, 106 / \mathrm{min}$, and $4.3 \mathrm{~g} / \mathrm{dL}$ respectively. In physical examination, patient was observed to have fatique with tachypnea, tachycardia, and peripheral findings of liver cirrhosis. There was smoking history of 40 package/year and Diabetes mellitus (DM). Upper gastrointestinal system (GIS) endoscopy was carried out urgently and tortuous and communicating varices with active bleeding at grade III and positive red spot signs were detected (Fig.1).

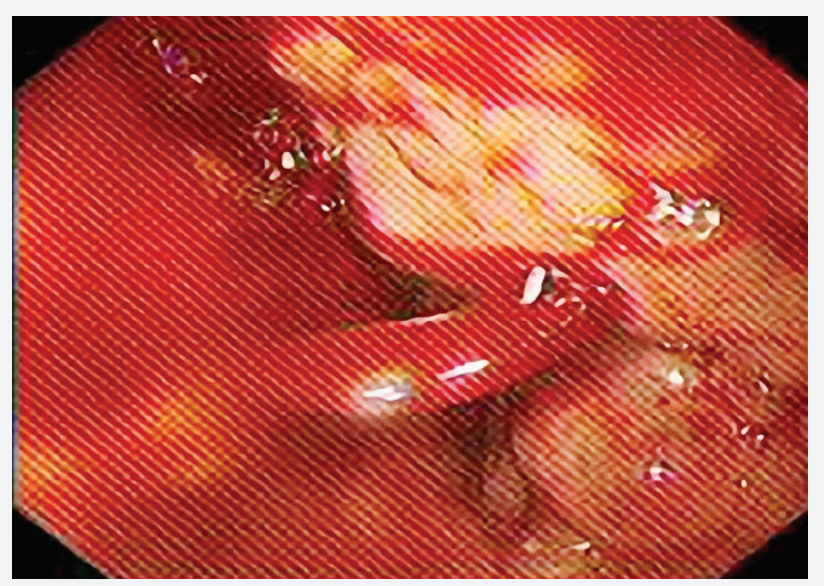

Fig. 1. Active variceal bleeding. 
There was no varices in the stomach. Band ligation was carried out, and medical treatment commented. As terlipressin was not available, octrotide was used as vasopressor agent. However, due to recurrent abundant variceal bleeding, alcohol injection was made to varices with a second endoscopy. But, bleeding recurred about 24 hours later. After the third endoscopy sudden distension and deterioration of general condition occurred. Emergency laparotomy was carried out in general surgery department with the suspicion of perforation. No perforation was observed in operation and procedure was finished after administering sclerotherapy to the varices. Bleeding was controlled. In the operation, a mass lesion at the size of $4 \times 5 \mathrm{~cm}$ was seen in segment 3 of liver and biopsy sample was obtained. Pathology results were found to be compatible with HCC.

Post-operative bleeding did not occur and oral nutrition was initiated but there was increase in ascites. For draining, paracentesis was carried out many times. Spontaneous bacterial peritonitis developed. Pseudomonas putida growth was seen in culture. It was sensitive to sulperazon and controlled with treatment. Nevertheless, abundant variceal bleeding recurred band ligation was carried out two times again and sclerotherapy performed with polidocanol (2\% aethoxysklerol ) and $70 \%$ alcohol. The severity of bleeding decreased, but it could not be entirely controlled. Daily erithrocyte support was carried out. In the sixth endoscopy procedure, healing ulcers were observed in the lower end of esophagus. Bleeding in the form of oozing was seen in this region. No additional intervention was made. Paracentesis was made and simultaneously human albumin was infused. Hepatic encephalopathy developed. Extended-spectrum betalactamase $(\mathrm{ESBL})(+)$ Klebsiella growth occurred in urine culture. Hepatic encephalopathy was treated by using ertapenem. On the 48th day of hospitalization, 13 days after the last dose of octreotide, a sudden chest pain developed. ST segment elevations were seen at the inferolateral derivations of the electrocardiogram (ECG).

The patient was transferred to catheter laboratory of cardiology department with the diagnosis of acute infero-lateral myocardial infarction. (Fig. 2 a,b,c).

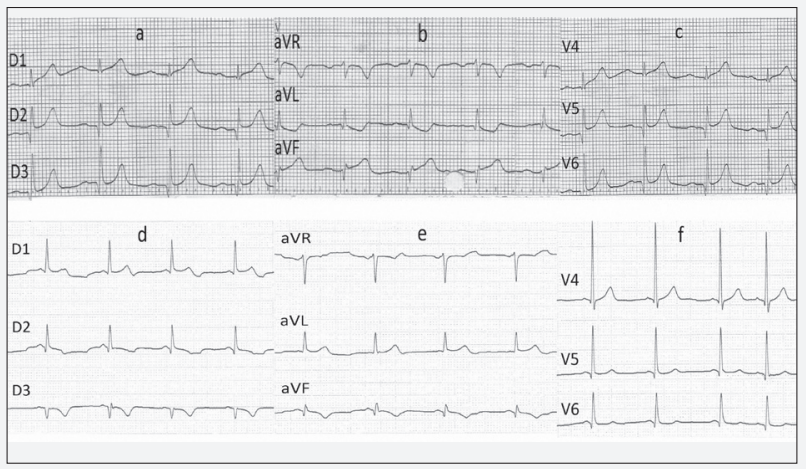

Fig. 2. Marked ST-segment elevations (a, b, c) and resolutions (d, e, f) in leads (D2-D3, aVF and V4-V5-V6) before and after stent implantation, respectively.

Total occlusion was detected in circumflex artery and percutan transluminal coronary angioplasty (PTCA) was performed and then two stents were placed. Distal blood flow was obtained (Fig. 3,4). No significant lesion was observed in left anterior descending (LAD) and right coronary (RCA)

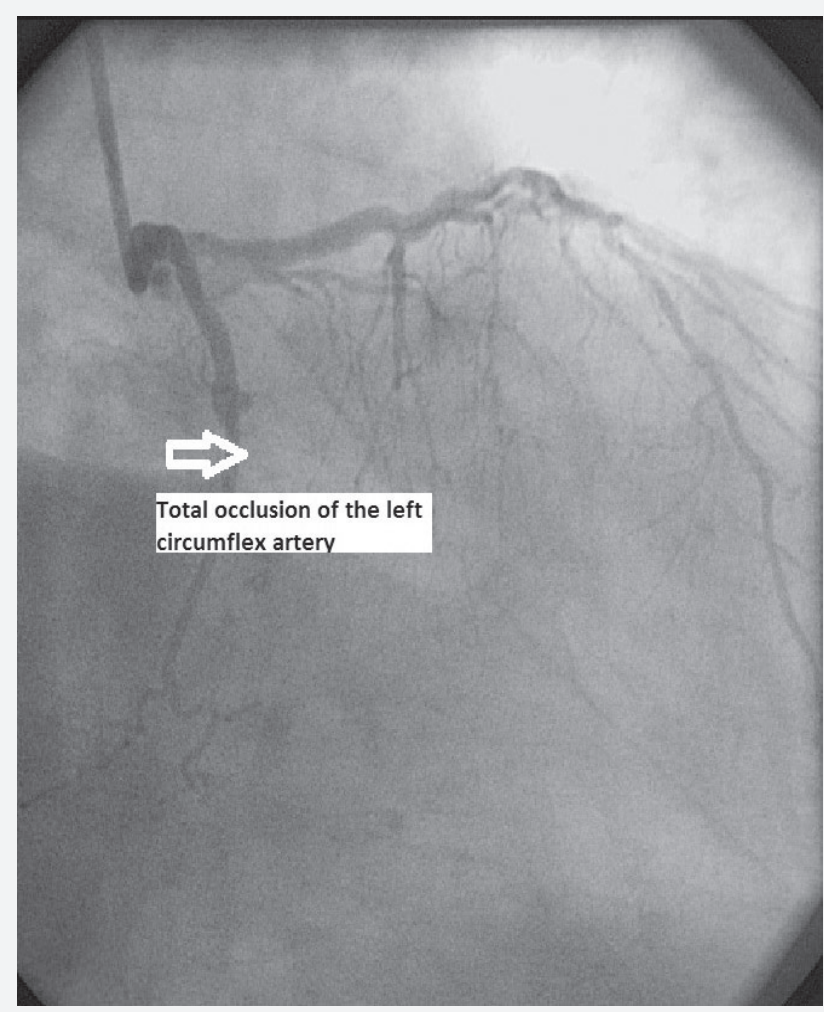

Fig. 3. Coronary angiogram demonstrates total occlusion of the left circumflex artery.

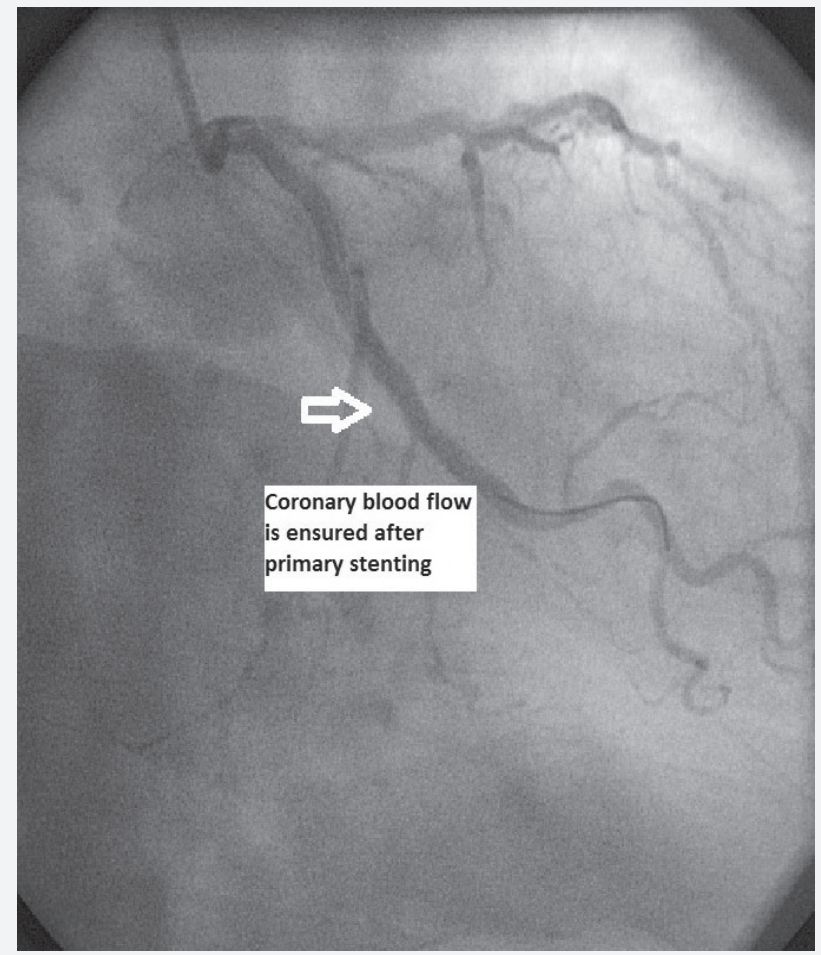

Fig. 4. The view of coronary angiography after stent implantation.

arteries. ST resolution was seen (Fig. 2 d,e,f) and chest pain was relieved. Aspirin and clopidogrel were initiated.

The patient was hospitalized overall for 55 days and during this time, he received totally 62 units erithrocyte and fresh whole blood, 59 units fresh frozen plasma and 11 units thrombocytes. Hgb value was maintained at 8-9 g/dL. In spite 
of this challenging process, he was discharged in a satisfactory general condition with his bleeding controlled.

\section{Discussion}

One of the most important causes of morbidity and mortality associated with cirrhosis is variceal bleeding (Garcia-Tsao and Bosch, 2010). Each variceal bleeding is associated with $30 \%$ of mortality rate (Smith and Graham, 1982).

In acute esophageal variceal bleeding, early treatment with vasoactive drugs such as terlipressin, somatostatin and octreotide may be life saving. These drugs decrease blood flow towards portal vein via their vasoconstructive effects in dilated splanchnic vessels and decrease portal pressure.

Acute myocardial infarction usually arises in the presence of atherosclerotic risk factors such as advanced age, smoking, and Diabetes mellitus and of coronary artery disease. Myocardial infarction (MI) may develop in many other conditions without coronary atherosclerosis. One of these conditions is the vasospasm in coronary arteries. Two cases of MI associated with terlipressine have been reported in the literature (Rosario et al.,1996; Lee et al., 2004). Although octreotide was used in our case, long duration of the time between its use and MI suggests that marked impairment in metabolic balance leds to the progression of present atherosclerotic disease and hence MI (Lee et al.,2004). The fact that esophageal varice bleeding is a problem bringing about many negative complications and requires multidisciplinary approach is stressed once more in view of the above mentioned case.

\section{REFERENCES}

Dave, P., Romeu, J., Messer, J.,1983.Upper gastrointestinal bleeding in patients with portal hypertension: A reappraisal. J. Clin. Gastroenterol. $5,113-115$.

Garcia-Tsao, G., Bosch, J. N., 2010. Management of varices and variceal hemorrhage in cirrhosis. Engl J Med. 362, 823-832.

Lee, M.Y., Chu, C.S., Lee, K.T., Lee H.C., Su, H.M., Cheng, K.H., Sheu, S.H., Kaohsiung, W.L., 2004. Terlipressin-related acute myocardial infarction: A case report and literature review. J. Med. Sci. 20, 604-608.

Odelowo, O.O., Smoot, D.T., Kim, K., 2002. Upper gastrointestinal bleeding in patients with liver cirrhosis. J. Natl. Med. Assoc. 94, $712-715$.

Rosario R., Lalanne B., Lebre P., Lepesan, D., Martelet, J.P., Dupont, M., Camatte, R., Lambot, G., Bourlière, M., 1996. Myocardial infarction after injection of terlipressin for digestive hemorrhage. Gastroenterol. Clin. Biol. 20, 712-713.

Smith, J.L., Graham, D.Y., 1982.Variceal hemorrhage: A critical evaluation of survival analysis. Gastroenterology. 82, 968-973. 